\title{
Abelhas Euglossina (Hymenoptera, Apidae) coletadas em uma monocultura de eucalipto circundada por Cerrado em Urbano Santos, Maranhão, Brasil
}

\author{
Fernanda N. Mendes, Márcia M. C. Rêgo \& Cristiane C. de Carvalho
}

Departamento de Biologia, Universidade Federal do Maranhão. Av. dos Portugueses, s/n, Campus Bacanga, 65080-580 São Luís, MA. (fnmodel@yahoo.com.br; regommc@uol.com.br; cristiane_c@yahoo.com.br)

\begin{abstract}
Euglossina bees (Hymenoptera, Apidae) collected in an eucalyptus monoculture surounded by Cerrado, Urbano Santos, MA, Brazil. Males of Euglossina bees were collected in benzil benzoate, eucaliptol, eugenol, methyl salicylate and vanillin scent baits in an eucalyptus monoculture surrounded by cerrado, located in the municipality of Urbano Santos, Maranhão. The collections were carried out monthly, from April 2001 to April 2002, between 8 a.m. and 4 p.m., totaling 96 hours of sampling, resulting in 58 individuals of 3 genera and 10 species. Euglossa Latreille, 1802 was the most abundant genus, followed by Eufriesea Cockerell, 1909 and Eulaema Lepeletier, 1841. The most frequent species were Euglossa cordata (Linnaeus, 1758), Euglossa gaianii Dressler, 1982 and Euglossa modestior (Dressler, 1982). Eucaliptol was the most attractive chemical bait. The highest frequencies of visits were in the morning and the highest abundance in September, in the drought period, and December, in the rainy period.
\end{abstract}

KEYWORDS. Euglossina, eucalyptus monoculture, abundance, diversity, scent baits.

RESUMO. Machos de Euglossina foram coletados por meio de iscas-odores de benzoato de benzila, eucaliptol, eugenol, salicilato de metila e vanilina em uma monocultura de eucalipto circundada por cerrado, no município de Urbanos Santos, Maranhão. As coletas foram realizadas mensalmente, de abril de 2001 a abril de 2002, entre $8 \mathrm{~h}$ e $16 \mathrm{~h}$, totalizando 96 horas de amostragem. Foram coletados 58 indivíduos de 3 gêneros e 10 espécies. Euglossa Latreille, 1802 foi o gênero mais abundante, seguido por Eufriesea Cockerell, 1909 e Eulaema Lepeletier, 1841. As espécies mais freqüentes foram Euglossa cordata (Linnaeus, 1758), Euglossa gaianii Dressler, 1982 e Euglossa modestior (Dressler, 1982). Eucaliptol foi a essência mais atrativa. As maiores frequiências de visitas ocorreram no período da manhã e as maiores abundâncias em setembro, no período de estiagem, e em dezembro, no período chuvoso.

PALAVRAS-CHAVE. Euglossina, monocultura de eucalipto, abundância, diversidade, iscas-odores.

As abelhas da Subtribo Euglossina forrageiam em uma grande diversidade de plantas, sendo consideradas importantes polinizadoras de algumas espécies de Orchidaceae, que são polinizadas exclusivamente por essas abelhas, as quais coletam substâncias odoríferas em suas flores (WiLliams, 1982; WiLliams \& WhitTEN, 1983). Adicionalmente, a grande diversificação biológica, abundância e importância destas abelhas para muitos ecossistemas - associados à atração e captura em iscasodoríferas - tornam-as possíveis indicadoras da qualidade ambiental de áreas naturais ou de conservação (BROwN JR., 1991).

Diante disso, as abelhas Euglossina são altamente adequadas para estudos das conseqüências diretas e indiretas da fragmentação florestal na estrutura e dinâmica das comunidades de fragmentos florestais (BECKER et al., 1991). O número de abelhas nas iscas reflete padrões de emergência e é correlacionado com a visita aos recursos naturais e, consequientemente, pode indicar a atual abundância de machos de Euglossina (ACKERMAn, 1983).

O Cerrado no Brasil destaca-se como o segundo bioma em extensão territorial, constituído por uma série de formações vegetais muito ricas. Porém são poucos os relatos sobre a fauna de Euglossina em áreas de domínio do Cerrado (RebÊlo \& CABral, 1997; RebÊlo \& GARÓFAlo, 1997). No Maranhão, as áreas de Cerrado vêm sendo continuamente devastadas e fragmentadas, dando lugar a monoculturas de eucalipto e soja, as quais não oferecem os recursos florais necessários ou sítios de nidificação essenciais para a reprodução das abelhas (MiLET-PinHEIRO \& SCHLINDWEIN, 2005).

As abelhas Euglossina são conhecidas por serem polinizadoras de longa distância em florestas tropicais, possuindo uma extensão de vôo de mais de $20 \mathrm{~km}$ (JANZEN, 1971; Ackerman \& Montalvo, 1985). Porém, existem controvérsias quanto à extensão de vôo entre fragmentos de florestas e a relação entre a área do fragmento e a abundância e riqueza de espécies (Powell \& Powell, 1987; BECKER et al., 1991; TONHASCA et al., 2003).

Neste contexto, objetivou-se identificar e analisar a composição de espécies da comunidade de abelhas Euglossina em uma monocultura de eucalipto inserida no Cerrado, com base nos aspectos de riqueza, abundância e diversidade.

\section{MATERIAL E MÉTODOS}

O estudo foi realizado no município de Urbano Santos, nordeste do Maranhão (312'28' S; 4324'12'”N), em 4 ha de uma monocultura de eucalipto circundada totalmente por vegetação de cerrado.

O clima da região é tropical megatérmico (enquadrando-se no tipo Aw', classificação de Köppen), com pluviosidade anual em torno de $1.800 \mathrm{~mm}$, sendo $89 \%$ desse total concentrado nos meses de dezembro a maio (e os meses de julho a outubro muito secos); a temperatura média anual varia entre $26^{\circ} \mathrm{C}$ e $27^{\circ} \mathrm{C}$. A vegetação predominante na região é o Cerrado, ocorrendo 
ainda formações de Floresta Estacional Semidecídua e manchas de vegetação mista, como a mata mesofítica (BRASIL, 1984).

Foram realizadas coletas mensais de abril de 2001 a abril de 2002, das 8 às $16 \mathrm{~h}$, totalizando 96 horas de amostragem. Empregou-se cinco substâncias aromáticas: benzoato de benzila, eucaliptol, eugenol, salicilato de metila e vanilina. Essas essências foram colocadas em chumaços de algodão envolvidos em gaze, amarrados com barbante e pendurados em um barbante maior, preso nos troncos dos eucaliptos; as iscas foram dispostas nesse barbante, entre os eucaliptos, a uma altura média de 1,5 $\mathrm{m}$ do solo e distantes $5 \mathrm{~m}$ umas das outras.

As abelhas atraídas foram capturadas com rede entomológica, colocadas em câmaras mortíferas e acondicionadas em sacos plásticos com etiquetas contendo data, tipo de substância odorífera utilizada e horário em que foram coletadas. Os exemplares foram identificados pelo Dr. José M. Macário Rebêlo, da Universidade Federal do Maranhão, e estão depositados na coleção entomológica do Laboratório de Estudos sobre Abelhas (LEA) do Departamento de Biologia da Universidade Federal do Maranhão.

Dados locais de temperatura e umidade relativa foram tomados nos dias de coleta, a cada hora, através de um termohigrômetro. Os dados referentes à precipitação foram obtidos na estação meteorológica na sede da Comercial e Agrícola Paineiras, a $5 \mathrm{~km}$ do local de estudo.

Foi utilizado o test $\mathrm{t}(\mathrm{p}<0,05)$ para avaliar as diferenças na proporção de machos capturados entre os períodos (matutino/vespertino; chuvoso/de estiagem). O coeficiente de correlação de Spearman (r) foi calculado para avaliar a relação entre o número de espécimes encontrados e as médias da temperatura, umidade relativa e precipitação. O índice de diversidade de ShannonWiener (H') (PIELOU, 1975) foi empregado para verificar a diversidade de abelhas na área estudada. Também foi feito o cálculo da porcentagem de similaridade para verificar a semelhança entre sítios na mesma localidade de estudo. Dados resultantes de dois outros sítios (4 ha de mata mesofítica, isolados por monoculturas de eucalipto, e 5 ha de mata ciliar) (CARVALHo et al., 2006) inventariados na mesma localidade e com metodologia de coleta semelhante ao descrito anteriormente, foram utilizados na discussão. As distâncias entre os pontos de coleta em cada sítio são: monocultura de eucaliptomata ciliar: $9,7 \mathrm{~km}$; monocultura de eucalipto-mata mesofítica: $17,9 \mathrm{~km}$; mata ciliar-mata mesofítica: $9,5 \mathrm{~km}$.

\section{RESULTADOS}

Foram coletados 58 machos distribuídos em três gêneros e dez espécies $\left(H^{\prime}=1,71\right)$, sendo a maioria (seis) do gênero Euglossa Latreille, 1802 (Tab. I). A espécie mais abundante foi Euglossa cordata (Linnaeus, 1758) $(39,6 \%$; n=23), seguida por Euglossa gaianii Dressler, 1982 (25,8\%; n=15) e Euglossa modestior Dressler 1982 $(12 \% ; \mathrm{n}=7)$.

As cinco essências utilizadas atraíram machos, sendo a mais atrativa o eucaliptol $(82,7 \% ; n=48)$ e as menos efetivas a vanilina e o salicilato de metila $(1,7 \%$; $\mathrm{n}=1$ ). $\mathrm{O}$ número total de machos coletados em cada isca odorífera e o total de iscas visitadas por cada espécie estão representados na tabela I.

Foram registradas visitas às iscas durante todo o período de coleta, principalmente das 9 às 10 horas, quando a temperatura média estava em torno de $29,5^{\circ} \mathrm{C}$. Nos horários seguintes houve queda na freqüência de visitas, que se manteve baixa por todo o período da tarde, com temperatura média próxima aos $31^{\circ} \mathrm{C}$ (Tab. II, Fig. 1).

Euglossa chalybeata Friese, 1925, E. townsendi Cockerell, 1904, Eufriesea mussitans (Fabricius, 1787), Eulaema cingulata (Fabricius, 1804) e E. nigrita Lepeletier, 1841 visitaram as iscas somente no período da manhã. Euglossa gaianii, E. cordata, E. modestior e Eufriesea nigrescens (Friese, 1925) também foram observadas durante a tarde, porém em baixa densidade. Euglossa melanotricha Moure, 1967 só foi observada no período entre 12 e 13 horas.

Considerando-se as espécies mais abundantes, Euglossa gaianii apresentou pico de abundância nos horários de 9 a 10 h e de 11 a 12 h, E. cordata entre 8 e 11 horas, e E. modestior das $10 \mathrm{~h}$ às $11 \mathrm{~h}$ (Tab. II). Houve diferença significativa $(\mathrm{t}=3,49 ; \mathrm{p}<0,05)$ entre o número de indivíduos amostrados visitando as iscas durante o período da manhã $(84,4 \%)$ e o período da tarde $(15,5 \%)$.

Tabela I. Número de machos de abelhas Euglossina atraídos por benzoato de benzila (BB), eucaliptol (EC), eugenol (EG), salicilato de metila (SM) e vanilina (VN), em Urbano Santos, Maranhão.

\begin{tabular}{|c|c|c|c|c|c|c|c|c|}
\hline Espécies & $\mathrm{BB}$ & $\mathrm{EC}$ & EG & SM & $\mathrm{VN}$ & Total & $\%$ & Total iscas \\
\hline Eufriesea nigrescens (Friese, 1925) & & 1 & 2 & & & 3 & 5,1 & 2 \\
\hline Eufriesea mussitans (Fabricius, 1787) & & & & 1 & & 1 & 1,7 & 1 \\
\hline Eulaema nigrita Lepeletier, 1841 & & 1 & & & & 1 & 1,7 & 1 \\
\hline Eulaema cingulata (Fabricius, 1804) & & & 2 & & 1 & 3 & 5,1 & 2 \\
\hline Euglossa cordata (Linnaeus, 1758) & 2 & 21 & & & & 23 & 39,6 & 2 \\
\hline Euglossa gaianii Dressler, 1982 & & 15 & & & & 15 & 25,8 & 1 \\
\hline Euglossa modestior Dressler, 1982 & 2 & 5 & & & & 7 & 12,0 & 2 \\
\hline Euglossa melanotricha Moure, 1967 & & 1 & & & & 1 & 1,7 & 1 \\
\hline Euglossa chalybeata Friese, 1925 & & 3 & & & & 3 & 5,1 & 1 \\
\hline Euglossa townsendi Cockerell, 1904 & & 1 & & & & 1 & 1,7 & 1 \\
\hline $\mathrm{N}^{\mathrm{o}}$ de espécies & 2 & 8 & 2 & 1 & 1 & & & \\
\hline \% dos indivíduos capturados & 6,8 & 82,7 & 6,8 & 1,7 & 1,7 & & & \\
\hline Total de indivíduos & 4 & 48 & 4 & 1 & 1 & 58 & & \\
\hline
\end{tabular}


As maiores freqüências ocorreram nos meses de setembro - no período de estiagem - e dezembro, no período chuvoso; a maior diversidade também foi observada no mês de dezembro, havendo alta concentração de espécies no período chuvoso $(\mathrm{n}=8)$ (Tab. III, Fig. 2). Em relação ao número de indivíduos e de espécies, não houve diferença significativa entre os períodos chuvoso e de estiagem (número de indivíduos: $\mathrm{t}=-1,08 ; \mathrm{p}<0,05 /$ número de espécies: $\mathrm{t}=-1,58 ; \mathrm{p}<0,05)$.

Cinco espécies foram capturadas somente no período chuvoso: Euglossa chalybeata, E. melanotricha, E. townsendi, Eufriesea nigrescens e E. mussitans. Eulaema nigrita só foi observada no período de estiagem; as demais espécies ocorreram em ambos os períodos, sendo que destas E. gaianii e E. cordata foram mais freqüentes no período chuvoso (Tab. III).

Comparando-se a flutuação mensal das abelhas com a variação dos fatores ambientais (Fig. 3) foram observadas correlações positivas entre: número de indivíduos e temperatura $(\mathrm{r}=0,98 ; \mathrm{p}<0,05)$, indivíduos e umidade $(\mathrm{r}=0,59$; $\mathrm{p}<0,05)$, número de espécies e temperatura $(r=0,99 ; \mathrm{p}<0,05)$ e espécies e umidade $(r=0,61 ; \mathrm{p}<0,05)$. Não houve correlação entre número de indivíduos e precipitação, nem entre quantidade de espécies e precipitação.

\section{DISCUSSÃO}

Quanto à composição da fauna de abelhas Euglossina, resultados semelhantes aos encontrados neste trabalho foram observados por M. H. M. Magalhães (dados inéditos) em São Benedito do Rio Preto, município vizinho de Urbano Santos, em área de mata ciliar (também no bioma cerrado): seis espécies de Euglossa, duas de Eulaema, duas de Eufriesea e uma de Exaerete (Tab. IV). Apesar de nenhum indivíduo deste último gênero ter sido coletado na monocultura de eucalipto, foi observado um espécime em campo.

Comparativamente ao estudo realizado em dois outros sítios (mata ciliar e mata mesofítica) no mesmo município (CARVALHO et al., 2006), o número de espécies amostrados na monocultura de eucalipto $(n=10)$ foi superior ao encontrado na mata mesofítica $(n=7)$ e inferior ao da mata ciliar ( $n=16$ ) (Tab. IV). De acordo com a composição de espécies, as áreas da mata mesofítica e
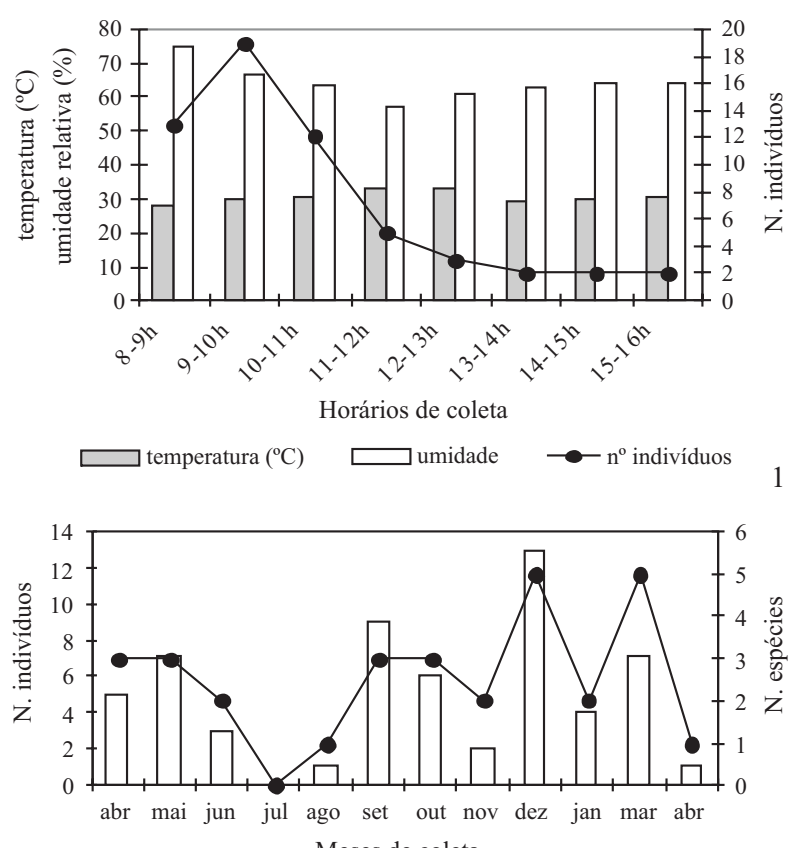

Meses de coleta

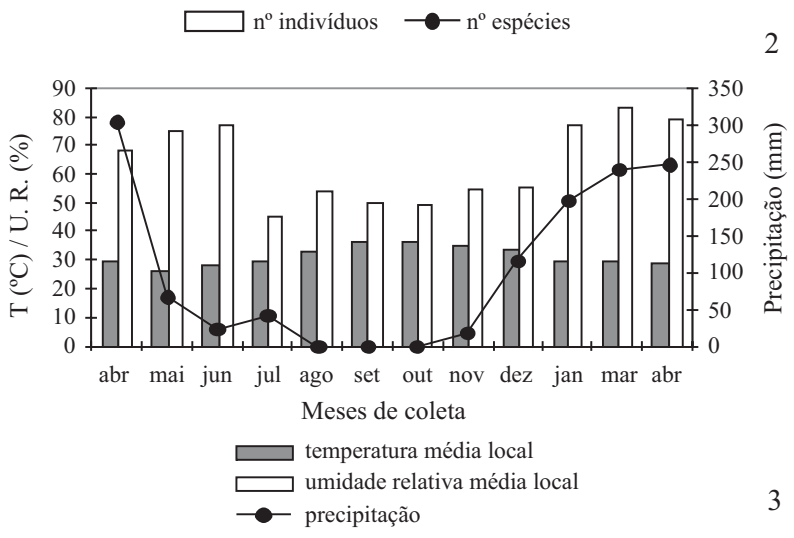

Figs. 1-3. 1, Número de indivíduos capturados, temperatura e umidade relativa médias por horário de coleta; 2 , número mensal de indivíduos e de espécies coletados; 3 , dados climáticos mensais (temperatura, umidade relativa e precipitação), de abril de 2001 a abril de 2002, Urbano Santos, Maranhão, Brasil.

Tabela II. Número de machos de abelhas Euglossina capturados das 8 às 16h, de abril de 2001 a abril de 2002, em Urbano Santos, Maranhão, Brasil.

\begin{tabular}{|c|c|c|c|c|c|c|c|c|c|}
\hline Espécies/ Horários & $8-9 \mathrm{~h}$ & $9-10 \mathrm{~h}$ & $10-11 \mathrm{~h}$ & $11-12 \mathrm{~h}$ & $12-13 \mathrm{~h}$ & $13-14 \mathrm{~h}$ & $14-15 \mathrm{~h}$ & $15-16 \mathrm{~h}$ & Total \\
\hline Euglossa chalybeata Friese, 1925 & & 1 & 1 & 1 & & & & & 3 \\
\hline Euglossa gaianii Dressler, 1982 & 2 & 6 & 1 & 4 & 1 & 1 & & & 15 \\
\hline Euglossa cordata (Linnaeus, 1758) & 7 & 7 & 6 & & 1 & 1 & & 1 & 23 \\
\hline Euglossa modestior Dressler, 1982 & & 2 & 3 & & & & 1 & 1 & 7 \\
\hline Euglossa melanotricha Moure, 1967 & & & & & 1 & & & & 1 \\
\hline Euglossa townsendi Cockerell, 1904 & & 1 & & & & & & & 1 \\
\hline Eufriesea nigrescens (Friese, 1925) & & 1 & 1 & & & & 1 & & 3 \\
\hline Eufriesea mussitans (Fabricius, 1787) & & 1 & & & & & & & 1 \\
\hline Eulaema cingulata (Fabricius, 1804) & 3 & & & & & & & & 3 \\
\hline Eulaema nigrita Lepeletier, 1841 & 1 & & & & & & & & 1 \\
\hline $\mathrm{N}^{\circ}$ espécies & 4 & 7 & 5 & 2 & 3 & 2 & 2 & 2 & \\
\hline Total indivíduos & 13 & 19 & 12 & 5 & 3 & 2 & 2 & 2 & 58 \\
\hline
\end{tabular}


Tabela III. Número mensal de machos de abelhas Euglossina capturados de abril de 2001 a abril de 2002, em Urbano Santos, Maranhão.

\begin{tabular}{|c|c|c|c|c|c|c|c|c|c|c|c|c|c|}
\hline Anos & & & & & 200 & & & & & & 2002 & & \\
\hline Períodos & & & & & & gem & & & & & uvos & & Total \\
\hline Espécies/ Meses & A & M & $\mathrm{J}$ & $\mathrm{J}$ & A & $\mathrm{S}$ & $\mathrm{O}$ & $\mathrm{N}$ & $\mathrm{D}$ & $\mathrm{J}$ & M & A & \\
\hline Euglossa chalybeata Friese, 1925 & 2 & & & & & & & & & & & 1 & 3 \\
\hline Euglossa gaianii Dressler, 1982 & & 5 & & & & 1 & 2 & & 3 & 2 & 2 & & 15 \\
\hline Euglossa cordata (Linnaeus, 1758) & 1 & 1 & 2 & & & 5 & 3 & & 7 & 2 & 2 & & 23 \\
\hline Euglossa modestior Dressler, 1982 & & 1 & & & 1 & 3 & & 1 & 1 & & & & 7 \\
\hline Euglossa melanotricha Moure, 1967 & & & & & & & & & & & 1 & & 1 \\
\hline Euglossa townsendi Cockerell, 1904 & & & & & & & & & & & 1 & & 1 \\
\hline Eufriesea nigrescens (Friese, 1925) & 2 & & & & & & & & & & 1 & & 3 \\
\hline Eufriesea mussitans (Fabricius, 1787) & & & & & & & & & 1 & & & & 1 \\
\hline Eulaema cingulata (Fabricius, 1804) & & & 1 & & & & & 1 & 1 & & & & 3 \\
\hline Eulaema nigrita Lepeletier, 1841 & & & & & & & 1 & & & & & & 1 \\
\hline $\mathrm{N}^{\circ}$ espécies & 3 & 3 & 2 & 0 & 1 & 3 & 3 & 2 & 5 & 2 & 5 & 1 & \\
\hline Total indivíduos & 5 & 7 & 3 & 0 & 1 & 9 & 6 & 2 & 13 & 4 & 7 & 1 & 58 \\
\hline
\end{tabular}

Tabela IV. Riqueza e diversidade de machos de abelhas Euglossina coletados em duas localidades no Nordeste do Maranhão, Brasil (MC, mata ciliar; MM, mata mesofítica (CARVAlHo et al., 2006); ME, monocultura de eucalipto (este trabalho); SBRP, São Benedito do Rio Preto (M. H. M. Magalhães, dados inéditos)).

\begin{tabular}{|c|c|c|c|c|}
\hline Espécies & MC & MM & ME & SBRP- MC \\
\hline Eufriesea surinamensis (Linnaeus, 1758) & 3 & 1 & 1 & 2 \\
\hline Eufriesea nigrescens (Friese, 1925) & 3 & 1 & 3 & \\
\hline Eufriesea laniventris (Ducke 1902) & & & & 6 \\
\hline Eufriesea ornata (Mocsary,1896) & 2 & & & \\
\hline Eufriesea pulchra (Smith,1854) & 5 & & & \\
\hline Euglossa avicula Dressler,1982 & 1 & & & \\
\hline Euglossa chalybeata Friese, 1925 & 7 & & 3 & 1 \\
\hline Euglossa cordata (Linnaeus, 1758) & 54 & 16 & 23 & 24 \\
\hline Euglossa gaianii Dressler, 1982 & 18 & 5 & 15 & \\
\hline Euglossa liopoda Dressler, 1982 & 4 & & & \\
\hline Euglossa melanotricha Moure, 1967 & 1 & & 1 & \\
\hline Euglossa modestior Dressler, 1982 & 77 & 3 & 7 & 4 \\
\hline Euglossa pleosticta Dressler, 1982 & 1 & & & \\
\hline Euglossa securigera Dressler 1982 & & & & 2 \\
\hline Euglossa stilbonota Dressler 1982 & & & & 1 \\
\hline Euglossa truncata Rebêlo \& Moure,1995 & 1 & & & \\
\hline Euglossa townsendi Cockerell, 1904 & & & 1 & 1 \\
\hline Eulaema cingulata (Fabricius, 1804) & 52 & 8 & 3 & 40 \\
\hline Eulaema nigrita Lepeletier, 1841 & 26 & 4 & 1 & 8 \\
\hline Exaerete smaragdina (Guérin-Menéville,1845) & 28 & & 4 & \\
\hline $\mathrm{N}^{\mathrm{o}}$ indivíduos & 283 & 38 & 58 & 93 \\
\hline $\mathrm{N}^{\mathrm{o}}$ espécies & 16 & 7 & 10 & 11 \\
\hline $\mathrm{H}^{\prime}$ & 2,04 & 1,58 & 1,71 & 1,68 \\
\hline
\end{tabular}

da monocultura de eucalipto apresentaram maiores coeficiente e porcentagem de similaridade (Q.S. $=0,87$; P.S.=0,74), significando uma maior semelhança entre as comunidades de Euglossina nessas duas áreas. Já entre a mata ciliar e a monocultura de eucalipto a similaridade foi menor (Q.S.=0,72; P.S.=0,49), assim como entre as matas ciliar e mesofítica (Q.S.=0,61; P.S.=0,49). Talvez fatores como o tamanho das três áreas, a influência da vegetação do entorno (CARVALHo et al., 2006) e o isolamento da mata mesofítica quando comparada às outras áreas, possa ser considerado, entretanto são necessários estudos e testes mais quantitativos.

Em diversos trabalhos realizados (BRAGA, 1976; RaW, 1989; Morato et al., 1992; NeVES \& VianA, 1997;
Rebêlo \& Cabral, 1997; Rebêlo \& Garófalo, 1997; Albuquerque et al., 2001; Brito \& RÊGo, 2001; Sofia \& SuZuKi, 2004; CARvalho et al., 2006), eucaliptol também foi o composto que obteve o maior número de visitas. De acordo com SOFIA \& SUZUKI (2004), o eucaliptol apresenta maior volatilidade (baixo peso molecular) quando comparado a outros compostos aromáticos.

Euglossa chalybeata, E. gaianii, E. melanotricha, E. townsendii e Eulaema nigrita visitaram somente o eucaliptol. Porém, em Alcântara, Maranhão, Euglossa chalybeata, E. gaianii e Eulaema nigrita visitaram, além do eucaliptol, iscas de salicilato de metila e vanilina (BRITO \& RÊGO, 2001); e em São Benedito do Rio Preto, também no Maranhão, Euglossa townsendii foi coletada em isca 
de eugenol (M. H. M. Magalhães, dados inéditos). De acordo com REBÊLo (2001), uma sustância dificilmente é específica para uma espécie em particular, visto que compostos que atraem apenas uma ou nenhuma espécie em um local podem atrair várias em outro. $\mathrm{O}$ mesmo pode acontecer em relação às estações do ano, visto que as respostas dos machos a certas substâncias aromáticas estão sujeitas a variações geográficas e sazonais (REBÊLO, 2001). Isso pode explicar as diferentes respostas às iscas.

Salicilato de metila e vanilina foram compostos menos efetivos, ao contrário do que foi observado na Bacia Amazônica (Morato et al., 1992; Morato, 1994) e na Bahia (NEVES \& VIANA, 1997) onde salicilato foi bastante atrativo e em Viçosa, Minas Gerais (Peruquetti et al., 1999), em que vanilina foi o composto que atraiu o maior número de espécimes.

Duas hipóteses são sugeridas para o fato de alguns compostos considerados bons atrativos não atraírem Euglossina em determinados locais: (1) estes compostos aromáticos não estariam presentes em nenhum recurso natural utilizado pelos machos para obtenção de fragância, não sendo assim reconhecidos (PeruQuetTI \& CAMPos, 1997; Peruquetti, 1998); ou (2) estes compostos estariam ausentes nos materiais utilizados pelas fêmeas para a construção do ninho (PERUQUETTI et al., 1999).

A atividade diária dos machos de Euglossina em Urbano Santos apresentou-se, de modo geral, conforme o padrão descrito na literatura (Powell \& Powell, 1987; BRITO \& RÊGO, 2001), caracterizado pela maior freqüência de visitas durante a manhã, período caracterizado por temperaturas mais amenas. A redução na atividade dos machos observada após as 14 horas pode refletir a existência de um ritmo intrínseco dos machos, que determina suas atividades diárias na exploração de compostos aromáticos no ambiente (SANTOS \& SoFIA, 2002).

Uma correlação positiva entre o número de indivíduos coletados e a temperatura e umidade do ambiente também foi observada no sudeste do Brasil (RebÊlo \& Garófalo, 1991) e na Paraíba (BEZERra \& MARTINS, 2001).

Euglossa cordata, que foi a espécie mais abundante, é comumente encontrada o ano inteiro, de acordo com Rebêlo \& Silva (1999) e RebÊLo \& CABRAL (1997); em Urbano Santos, 56,5\% dos 23 indivíduos capturados ocorreram no período chuvoso. Já Euglossa modestior (terceira espécie mais abundante), foi mais observada no período de estiagem $(71,4 \%)$. Esta última espécie mostrou evidências de sazonalidade em Alcântara, sendo ativa em abril e agosto (final das chuvas e início do período de estiagem) (BRITO \& RÊGO, 2001).

$\mathrm{O}$ gênero Eufriesea inclui abelhas tipicamente sazonais, sendo algumas espécies ativas apenas durante um ou dois meses por ano (DRESSLER, 1982). Isso poderia explicar a ocorrência de E. nigrescens e E. mussitans apenas na estação chuvosa.

Devido à baixa densidade de indivíduos e à distribuição irregular ao longo do ano, não é seguro inferir sobre o padrão sazonal da maioria das espécies observadas.

Quando comparados os três habitats estudados no cerrado de Urbano Santos, percebe-se a baixa abundância dos Euglossina para uma área isolada de uma cultura exótica, como é o caso do eucalipto. A monocultura e a consequiente ausência de substratos para nidificação certamente afetam as populações de abelhas. A presença de corredores ecológicos ligando essas áreas às matas adjacentes (principalmente matas ciliares, as quais funcionariam como berçários naturais por sua variedade de flora e de nichos para nidificação) seria uma alternativa para minimizar o rareamento de espécies, garantindo a biodiversidade e a própria manutenção do ecossistema, uma vez que estas e outras abelhas são importantes agentes polinizadores.

Agradecimentos. Esta pesquisa recebeu apoio logístico, em Urbano Santos, da Comercial \& Agrícola Paineiras. Ao Prof. Dr. José Manoel Macário Rebêlo (Universidade Federal do Maranhão), pela identificação das abelhas; aos consultores anônimos, pelas valiosas críticas e sugestões.

\section{REFERÊNCIAS BIBLIOGRÁFICAS}

Ackerman, J. D. 1983. Diversity and seasonality of male Euglossina bees (Hymenoptera, Apidae) in central Panama. Ecology 64(2):274-283.

Ackerman, J. D. \& Montalvo, A. M. 1985. Longevity of euglossine bees. Biotropica 17:79-81.

Albuquerque, P. M. C.; Ferreira, R. G.; Rêgo, M. M. C.; Santos, C. S. \& BRito, C. M. S. 2001. Levantamento da fauna de abelhas silvestres (Hymenoptera, Apoidea) na região da "Baixada Maranhense": Vitória do Mearim, MA, Brasil. Acta Amazonica 31(3):419-430.

Becker, P.; Moure, J. S. \& Peralta, F. J. A. 1991. More about Euglossina bees in Amazonian Forest Fragments. Biotropica 23:586-591.

Bezerra, C. P. \& Martins, C. F. 2001. Diversidade de Euglossinae (Hymenoptera, Apidae) em dois fragmentos de Mata Atlântica localizados na região urbana de João Pessoa, Paraíba, Brasil. Revista Brasileira de Zoologia 18:823-835.

Braga, P. I. S. 1976. Atração de abelhas polinizadoras de Orchidaceae com auxílio de iscas-odores na campina, campinarana e floresta tropical úmida da Região de Manaus. Ciência e Cultura 28(7):767-773.

BRASIL. 1984. Diagnóstico dos principais problemas ambientais do Estado do Maranhão. São Luís, Lithograf. 194p.

Brito, C. M. S. \& RÊGo, M. M. C. 2001. Community of male Euglossina bees (Hymenoptera: Apidae) in a secondary forest, Alcântara, MA, Brazil. Brazilian Journal of Biology 61(4):631-638.

Brown JR., K. S. 1991. Conservation of neotropical environments: Insects as indicators, p. 349-404. In: Collins, N. M. \& Thomas, J. A. eds. The conservation of insects and their habitats. London, Academic. 430p.

Carvalho, C. C.; Rêgo, M. M. C. \& Mendes, F. N. 2006. Dinâmica de populações de Euglossina (Hymenoptera, Apidae) em mata ciliar, Urbano Santos, Maranhão, Brasil. Iheringia, Série Zoologia, 96(2):249-256.

Dressler, R. L. 1982. Biology of the Orchid Bees (Euglossina). Annual Review of Ecology and Systematics 13:373-394.

JANZEN, D. H. 1971. Euglossine bees as long distance pollinators of tropical plants. Science 171:203-205.

Milet-Pinheiro, P. \& Schlindwein, C. 2005. Do euglossine males (Apidae, Euglossini) leave a tropical rainforest to collect fragrances in sugarcane monocultures? Revista Brasileira de Zoologia 22(4):853-858.

Morato, E. F. 1994. Abundância e riqueza de machos de Euglossina (Hymenoptera: Apidae) em mata de terra firme e área de derrubada, nas vizinhanças de Manaus (Brasil). Boletim do Museu Paraense Emílio Goeldi 10(1):95-105.

Morato, E. F.; Campos, L. A. O. \& Moure, J. S. 1992. Abelhas Euglossina (Hymenoptera, Apidae) coletadas na Amazônia Central. Revista Brasileira de Entomologia 36(4):767-771.

Neves, E. L. Das \& Viana, B. F. 1997. Inventário da fauna de Euglossinae (Hymenoptera, Apidae) do Baixo Sul da Bahia, Brasil. Revista Brasileira de Zoologia 14(4):831-837. 
Peruquetti, R. C. 1998. Notes on adults of Euglossa townsendi (Apidae: Euglossina) reared from a trap nest. Anais da Sociedade de Entomologia do Brasil 27(2):309-311.

Peruquetti, R. C. \& Campos, L. A. O. 1997. Aspectos da biologia de Euplusia violaceae (Blanchard) (Hymenoptera, Apidae, Euglossina). Revista Brasileira de Zoologia 14(1):91-97.

Peruquetti, R. C.; Campos, L. A. O.; Coelho, C. D. P.; Abrantes, C. M. V. \& Lisboa, L. C. O. 1999. Abelhas Euglossini (Apidae) de áreas de Mata Atlântica: abundância, riqueza e aspectos biológicos. Revista Brasileira de Zoologia 16(Supl.2):101-108.

Pielou, E. C. 1975. Ecological diversity. New York, John Wiley \& Sons. 165 p.

Powell, A. H. \& Powell, G. V. N. 1987. Population dynamics of male euglossine bees Amazonian forest fragments. Biotropica 19(2): 176-179.

Raw, A. 1989. The dispersal of euglossine bees between isolated patches of eastern brazilian wet forest (Hymenoptera, Apidae). Revista Brasileira Entomológica 33(1):103-107.

RebêLo, J. M. M. 2001. História natural das Euglossíneas: as abelhas das orquídeas. São Luís, Lithograf. 152p.

RebêLo, J. M. M. \& Cabral, A. J. M. 1997. Abelhas Euglossinae de Barreirinhas, zona litoral da baixada oriental maranhense. Acta Amazonica 27(2):145-152.

RebêLo, J. M. M. \& Garófalo, C. A. 1991. Diversidade e sazonalidade de machos de Euglossini (Hymenoptera, Apidae) e preferências por iscas-odores em um fragmento de floresta no sudeste do Brasil. Revista Brasileira de Biologia 51:787-799.

1997. Comunidades de machos de Euglossina (Hymenoptera: Apidae) em matas semidecíduas do Nordeste do Estado de São Paulo. Anais da Sociedade Entomológica do Brasil 26(2):243-255.

RebÊLo, J. M. M. \& Silva, F. S. 1999. Distribuição das abelhas Euglossina (Hymenoptera, Apidae) no estado do Maranhão. Anais da Sociedade Entomológica do Brasil 28(3):389-401.

Santos, A. M. \& Sofia, S. H. 2002. Horário de atividade de machos de Euglossinae (Hymenoptera, Apidae) em um fragmento de floresta semidecídua no Norte do Estado do Paraná. Acta Scientiarum 24(2):375-381.

Sofia, S. H. \& Suzuki, K. M. 2004. Comunidades de machos de abelhas Euglossina (Hymenoptera: Apidae) em fragmentos florestais no sul do Brasil. Neotropical Entomology 33(6):693-702.

Tonhasca, A.; Albuquerque, G. S. \& Blackmer, J. L. 2003. Dispersal of euglossine bees between fragments of the Brazilian Atlantic Forest. Journal of Tropical Ecology 19:99-102.

Williams, N. H. 1982. The biology of orchids and euglossine bees. p. 119-171. In: ARditTI, J. ed. Orchid biology: Reviews and Perspectives. Ithaca, Cornell University.

Williams, N. H. \& WhitTEN, W. M. 1983. Orchid floral fragrances and male euglossine bees. Methods and advances in the last sesquidecade. Biological Bulletin 164:335-395. 